\title{
Identificação de propriedades de fluxo do caulim e dimensionamento de tremonha de silo para fluxo mássico
}

\section{Identification of flow properties of kaolin and hopper design for mass flow}

Fernando Pereira Mascarenhas ${ }^{1}$, Alexandre Luiz Amarante Mesquita ${ }^{1,2}$, Sérgio de Souza Custódio Filho ${ }^{1}$, André Luiz Amarante Mesquita ${ }^{2}$

\footnotetext{
${ }^{1}$ Faculdade de Engenharia Mecânica, Universidade Federal do Pará, Rua Augusto Corrêa 01, 66075-110, Belém, PA e-mail: nando280@hotmail.com; alexmesq@ufpa.br, engsergiocustodio@gmail.com

${ }^{2}$ Núcleo de Desenvolvimento Amazônico em Engenharia, Universidade Federal do Pará, 68.464-000 Tucuruí, PA e-mail: andream@ufpa.br
}

\section{RESUMO}

A transferência e armazenagem de caulim são etapas importantes para seu processo de beneficiamento. O projeto de um sistema para essas etapas necessita de um estudo detalhado do comportamento dinâmico do material granulado que se está utilizando. Problemas em silos podem ocorrer, em muitos casos, devido ao incorreto dimensionamento de sua geometria pela desconsideração das propriedades de fluxo do material granulado. Portanto, este trabalho se concentrou em analisar as propriedades de fluxo do caulim, com $4 \%$ de umidade, com o intuito de dimensionar uma tremonha cônica de um silo para fornecer um fluxo mássico (fluxo uniforme) ao material. Inicialmente, as propriedades de fluxo do caulim foram obtidas em laboratório. Em seguida, a partir da caracterização do caulim, o dimensionamento de uma tremonha cônica do silo foi realizado, por meio da teoria de Jenike, para determinação do ângulo de inclinação para fornecer o padrão de escoamento desejado. Em seguida, o diâmetro mínimo de descarga para evitar o arqueamento foi calculado por meio das teorias de Jenike e Enstad. Resultados experimentais de escoamento em silos de acrílico com tremonhas com diferentes configurações foram obtidos para validar os resultados teóricos. Os resultados dos ensaios experimentais apresentaram boa concordância com os resultados teóricos, os quais mostraram que o dimensionamento pela teoria de Jenike é mais conservador que a teoria de Enstad na determinação do diâmetro mínimo.

Palavras-chave: Silos, tremonha, fluxo mássico, caulim.

\section{ABSTRACT}

The transfer and storage of kaolin are important steps for its beneficiation process. The design of a system for such purposes requires a detailed study of the dynamic behavior of granular material that is being used. Problems can occur in silos, in many cases, due to incorrect design by not considering the granulated material flow properties. Therefore, this work has focused on analyzing the kaolin flow properties for particular moisture content $(4 \%)$ in order to design a conical hopper to provide a mass flow (uniform flow) pattern of the material. Initially, the kaolin flow properties were obtained from laboratory tests. Then, from the kaolin characterization, the design of a conical hopper was performed using Jenike theory in order to determine the angle that provides the desired flow pattern (mass flow). Next, the minimum discharge diameter to prevent flow obstruction (cohesive arching) was calculated by the theories of Jenike and Enstad. Experimental test results using acrylic silos with different configurations were obtained to validate the theoretical results. The results of experimental tests demonstrated good concordance with the theoretical results, which have shown that the design by Jenike theory is more conservative than the Enstad theory in determining the minimum diameter.

Keywords: Silo, hopper, mass flow, kaolin. 


\section{INTRODUÇÃO}

O caulim é formado basicamente pela caulinita (silicato hidratado de alumínio) e tem a cor branca por causa do seu baixo teor de ferro. Trata-se de um mineral argiloso branco, atóxico, com baixa condutividade térmica e elétrica e que não apresenta reatividade química [1]. Em geral, os caulins são minerais de origem residual, que derivam de rochas feldspáticas (como ocorre no Nordeste Brasileiro) ou também de origem sedimentar, onde o material encontra-se em seu estado mais puro (ocorrência típica no Nordeste do Pará, ao longo do Rio Capim) [2].

O caulim é um mineral estratégico para a balança comercial brasileira, tendo um relevante papel socioeconômico em vários estados [3,4]. O Brasil é o 5음 maior produtor de caulim do mundo, sendo que as maiores mineradoras do mineral se situam na Região Norte do País nos Estados do Pará e Amapá. O caulim exportado pelo Brasil é utilizado em diversos setores industriais, principalmente na indústria de papel (como elemento de alvura e fixação de impressão). No mercado interno é utilizado principalmente na fabricação de cimento e na indústria de cerâmica branca [4].

Uma das etapas do beneficiamento do caulim é o armazenamento deste material em silos. $\mathrm{O}$ incorreto dimensionamento do silo de armazenamento pode trazer vários problemas durante a descarga do material, tais como entupimento, segregação, falha estrutural, dentre outros [5]. Tipicamente, existem dois modos de padrão de fluxo em silos: fluxo mássico (ou de massa) e fluxo de funil [6]. No fluxo mássico, durante a descarga, a primeira porção que entra no silo é a primeira a sair, formando uma descarga uniforme. No fluxo de funil, parte do material é descarregada por meio de um canal de fluxo preferencial formado dentro do silo, enquanto que o restante do material permanece estacionário formando zonas de estagnação, que serão descarregadas por último. O fluxo mássico, que é um fluxo uniforme, é o tipo de fluxo preferencial nos projetos de silos. O fluxo de funil é a situação onde a maioria dos problemas em silos pode ocorrer [5].

O projeto tradicional de silos visando fluxo mássico foi proposto inicialmente por JENIKE [6], no qual se determina o ângulo de inclinação máximo para garantir o fluxo mássico, e em seguida determina-se a abertura mínima para a saída do material para que não haja a formação de arcos coesivos. A partir da abordagem de JENIKE, outros pesquisadores publicaram suas metodologias para o dimensionamento desse diâmetro mínimo $[7,8]$. Neste contexto, este trabalho focou na determinação das propriedades de fluxo do caulim com $4 \%$ de umidade, com o objetivo de dimensionar silos de acrílicos por meio das teorias de JENIKE [6] e ENSTAD [8], metodologias teóricas de dimensionamento bastante utilizadas até os dias atuais [9-12]. Para verificação dos resultados teóricos, foram realizados ensaios experimentais em laboratório com silos de acrílico para permitir a visualização do padrão do fluxo obtido.

\section{MATERIAIS E MÉTODOS}

\subsection{Caulim utilizado nos ensaios experimentais}

O material utilizado neste trabalho é o caulim com $4 \%$ de umidade já beneficiado na planta de Barcarena-PA da empresa Imerys (Figura 1). O Caulim da empresa Imerys é extraído das minas da empresa em Ipixuna do Pará às margens do Rio Capim e transportado por mineroduto até o beneficiamento na cidade de Barcarena, como mostra a Figura 2.

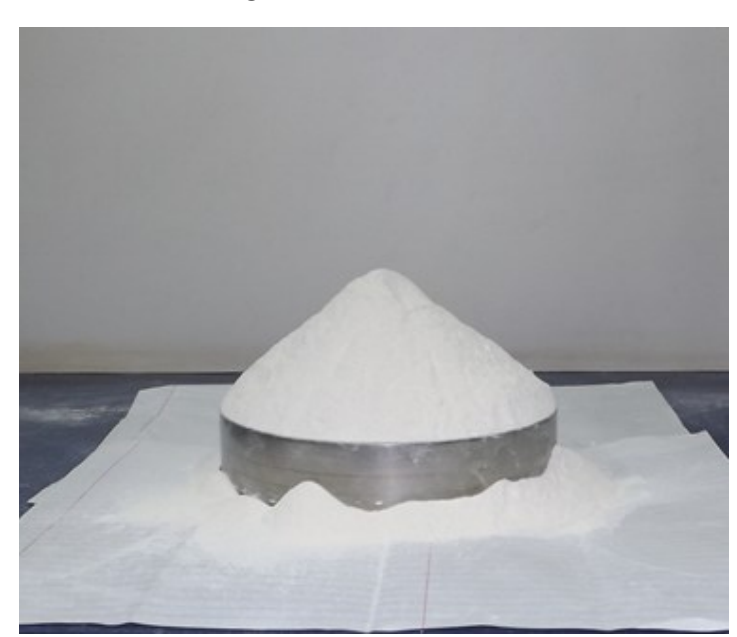

Figura 1: Caulim usado no trabalho.

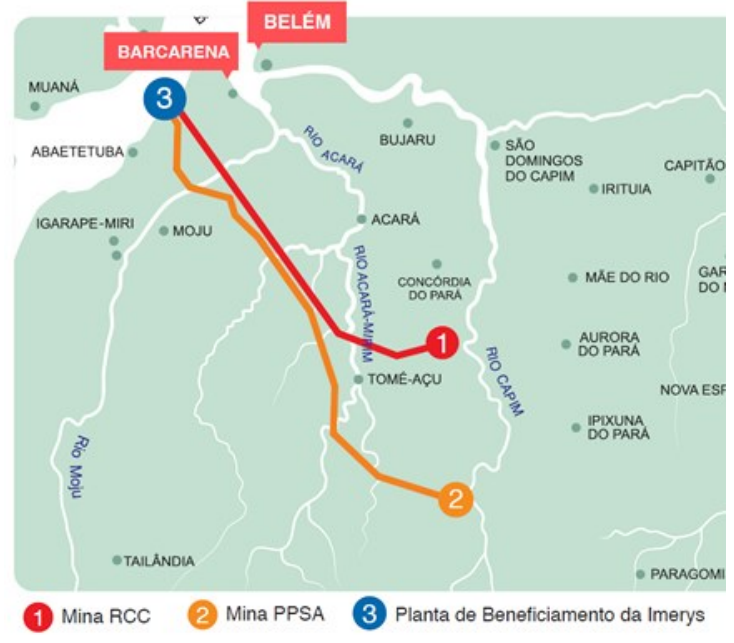

Figura 2: Locais das minas e usina de beneficiamento do caulim da Imerys [13]. 


\subsection{Determinação das propriedades de fluxo de material granulado}

Os equipamentos de medição usados para obtenção das propriedades de fluxo de materiais granulados são baseados nos equipamentos usados nos ensaios de cisalhamento da Mecânica dos Solos. Atualmente existem vários tipos de equipamentos para este fim, sendo que um dos mais utilizados é a Célula de Jenike (Figura 3). A célula Jenike é usada tanto para determinar as propriedades de fluxo do material, considerando o atrito entre material-material (Figura 3a) ou material-parede do equipamento de transporte ou armazenamento (Figura $3 b$ ). Os procedimentos para as realizações dos ensaios no equipamento estão descritos na norma D612897 ASTM [14].

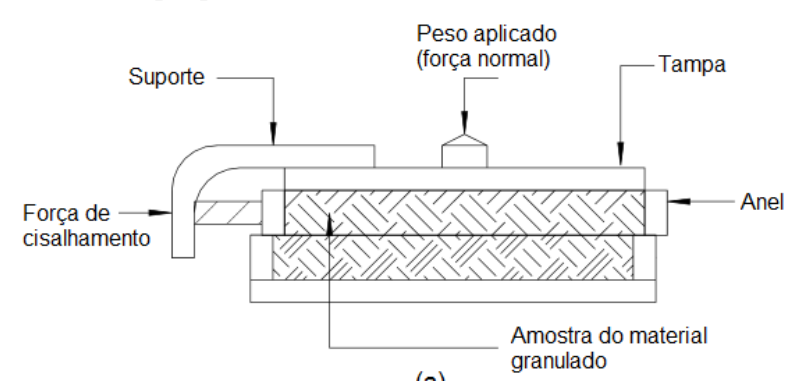

(a)

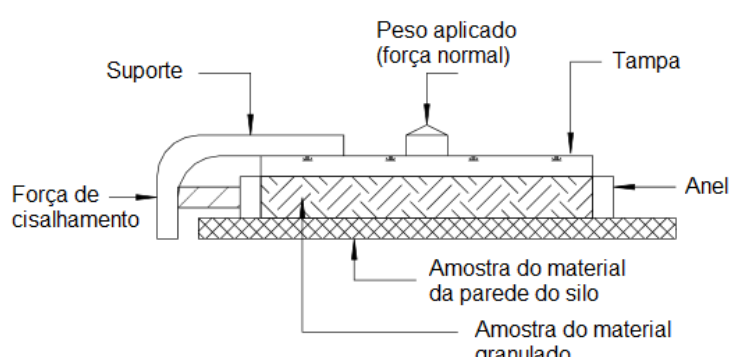

(b)

Figura 3: Célula de Jenike para ensaios de cisalhamento entre (a) material/material (b) e material/parede [15].

As principais propriedades de fluxo obtidas a partir dos testes de cisalhamento são a função de fluxo $(\mathrm{FF})$, ângulo de atrito interno efetivo $\left(\varphi_{\mathrm{e}}\right)$ e ângulo de atrito material-parede $\left(\varphi_{\mathrm{x}}\right)$. Tais propriedades são importantes no projeto de fluxo em silos. Para encontrar os níveis de tensão de cisalhamento onde ocorre o escoamento, estudos são feitos usando-se critérios de ruptura. Critérios de ruptura são formulações que procuram refletir as condições em que ocorre a ruptura dos materiais. Em mecânica dos solos, os critérios mais empregados são o Critério de Coulomb e o Critério de Mohr, que refletem bem o comportamento dos solos e são de aplicação relativamente simples. No critério de Mohr não ocorrerá ruptura enquanto o círculo representativo do estado de tensões (círculo de Mohr) se encontrar no interior de uma curva, que é a envoltória dos círculos relativos a estados de ruptura obtidos experimentalmente para o material [16]. A Figura 4 mostra a envoltória (ou curva de limite de escoamento - yield limit ou yield locus ou instantaneous yield locus) YL no gráfico tensão de cisalhamento $(\tau)$ versus tensão normal $(\sigma)$ [5]. Se o sólido granular estiver sob estado de tensão acima da envoltória, então irá ocorrer a falha e consequentemente o escoamento. Entretanto, abaixo da envoltória, o sólido não escoará.

Na teoria de JENIKE [6], é necessário conhecer as tensões cisalhantes necessárias para iniciar o escoamento. Isso significa identificar a Yield Locus (YL) no diagrama tensão cisalhante $(\tau)$ versus tensão normal $(\sigma)$. A YL é a curva tangente que envolve todos os círculos de Mohr que representam o estado de tensões em que o sólido inicia seu escoamento [6]. Vários círculos de Mohr podem ser desenhados tangentes a YL. Porém, para identificar as propriedades necessárias para projeto de silos são suficientes apenas dois círculos. $\mathrm{O}$ primeiro círculo de Mohr passa através da origem do diagrama $\tau$ versus $\sigma$ (Figura 4) cujo diâmetro é a tensão inconfinada (unconfined yield stress, $\sigma_{\mathrm{c}}$ ), uma medida de resistência relacionada ao estado de tensão onde o material não está confinado (contornos livres). O segundo círculo de Mohr é onde está localizado o ponto final da Yield Locus (end point, E), esse círculo representa o estado do material na tensão de consolidação $\sigma_{1}$.

Vale mencionar que as tensões normais nos círculos de Mohr para análises de sólidos granulados são positivas para tensões de compressão e tensões de cisalhamento são todas positivas. Por este motivo apenas os semicírculos positivos são plotados na análise.

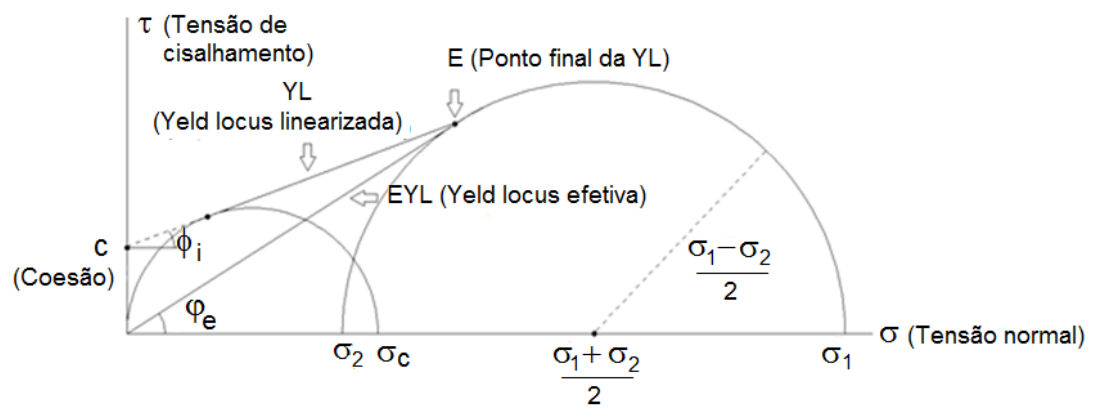

Figura 4: Curva de YL no círculo de Mohr. 
A Figura 4 mostra os dois semicírculos que definem $\sigma_{c}$ e $\sigma_{1}$. A YL tem forma curva, que por razões práticas a curva é aproximada por uma reta, sendo a inclinação da Yield Locus linearizada é o ângulo de atrito interno $\left(\varphi_{i}\right)$, e o ponto que a YL intercepta o eixo $\tau$ é a coesão, c.

Com os ensaios de cisalhamento realizados para várias tensões de pré-consolidação, têm-se várias curvas de YL e vários pares de pontos $\left(\sigma_{1}, \sigma_{c}\right)$. Então é possível obter em um gráfico a curva da tensão inconfinada em função da tensão de consolidação $\sigma_{c}\left(\sigma_{1}\right)$. Esta curva é denominada de Função de Fluxo (flow function - FF) (Figura 5). Vale ressaltar que quanto maior for a tensão de consolidação aplicada maior será a densidade do material granulado (pois, o material estará mais compactado) e também maior será a tensão não confinada. Com várias curvas de $\mathrm{YL}$, têm-se vários pontos finais $\mathrm{E}_{\mathrm{i}}$. A melhor reta que passa pela origem e pelos pontos $\mathrm{E}_{\mathrm{i}}$ é denominada linha de escoamento efetivo (effective yield locus), e é representada por EYL (Figura 6). O ângulo formado entre a EYL e a horizontal é o ângulo de atrito efetivo $\left(\varphi_{\mathrm{e}}\right)$ e caracteriza as condições de ruína para vários estados de pré-consolidação [16].

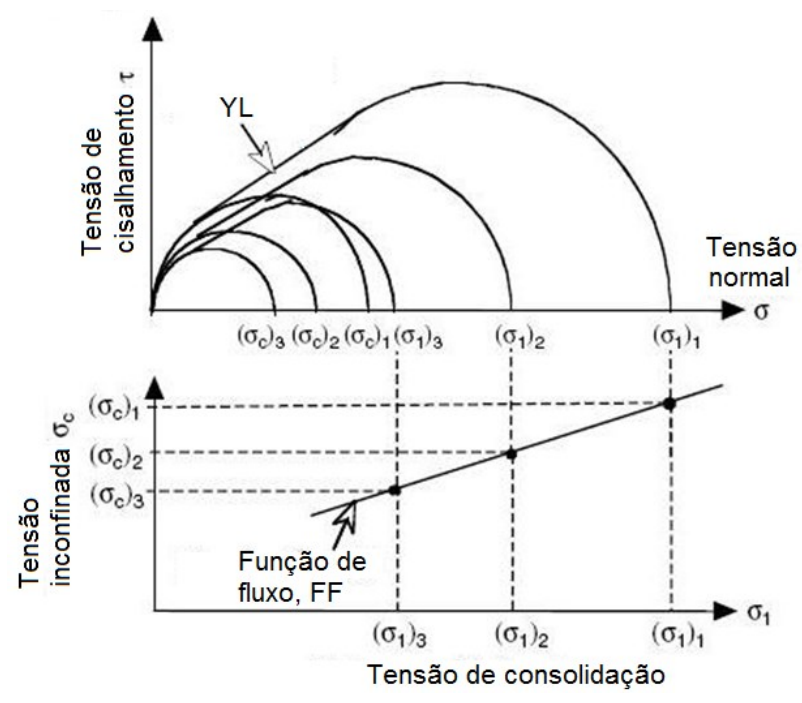

Figura 5: Família de YLs e função de fluxo FF [17].

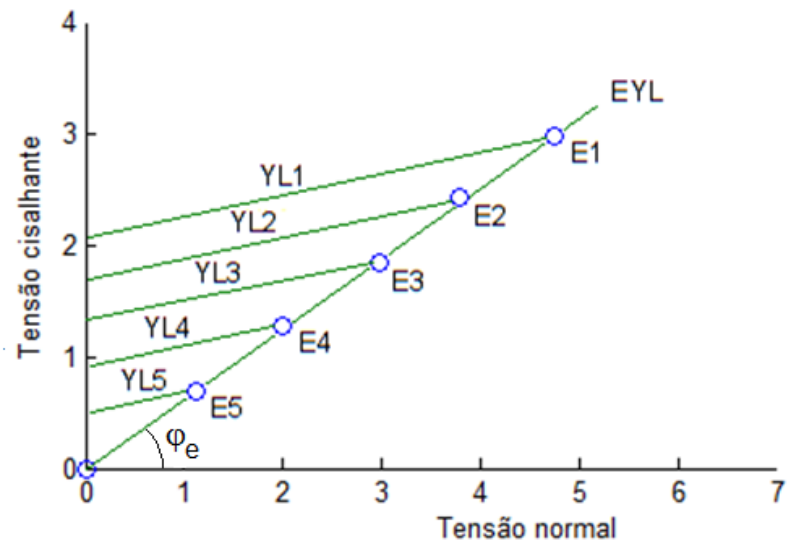

Figura 6: EYL e ângulo de atrito interno efetivo [16].

Atrito de parede é o atrito entre o material granulado e a superfície sólida do equipamento no qual o granulado é transportado. Na célula Jenike (Figura 3b), a amostra do material é submetida a uma tensão normal vertical que atua entre o material granulado e a superfície da amostra da parede, é a chamada tensão normal da parede $\left(\sigma_{\mathrm{w}}\right)$. Em seguida, a amostra do material é movida sobre a superfície da amostra da parede com velocidade constante $v$. Assim, é gerada a tensão de cisalhamento na parede $\left(\tau_{\mathrm{w}}\right)$ que vai aumentando até atingir um valor constante. Esta tensão de cisalhamento obtida, $\tau_{\mathrm{w} 1}$, é relativa à tensão normal aplicada, $\sigma_{\mathrm{w} 1}$. Em seguida, a tensão normal é diminuída e o procedimento repetido. Então, vários pares de valores de tensão normal de parede e tensão de cisalhamento de fluxo estável (regime permanente) são obtidos e plotados em um diagrama $\sigma_{\mathrm{w}}, \tau_{\mathrm{w}}$ (Figura 7). Os pares de valores $\sigma_{\mathrm{w}, \mathrm{i}}, \tau_{\mathrm{w}, \mathrm{i}}$ no diagrama $\sigma_{\mathrm{w}}, \tau_{\mathrm{w}}$ definem a curva (ou reta) que passa através desses pontos, denominada de lugar geométrico do deslizamento na parede (wall yield locus, WYL), como mostra a Figura 8. Caso a WYL seja uma curva ela deve ser linearizada e a inclinação da WYL será o ângulo de atrito com a parede $\varphi_{\mathrm{x}}$.

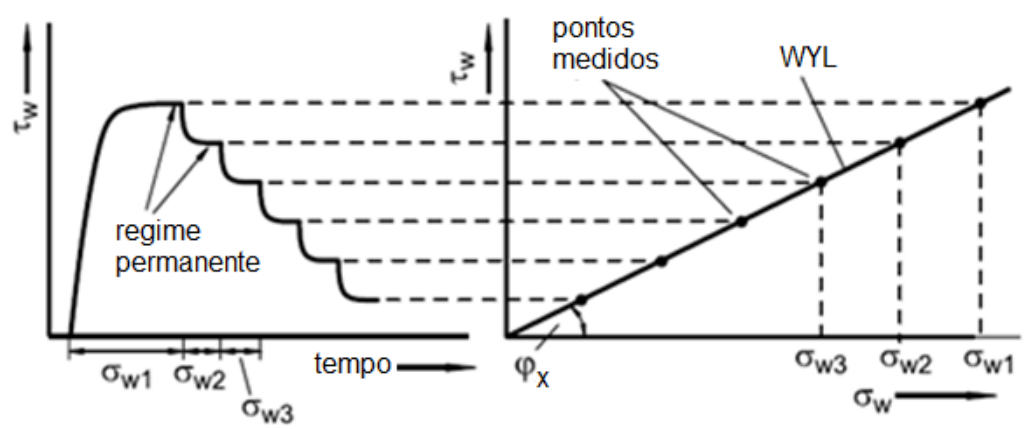

Figura 7: Curva WYL definindo o ângulo de atrito com a parede [5]. 


\subsection{Determinação do ângulo de inclinação de tremonhas cônicas de silos para fluxo mássico (teoria de Jenike)}

Tendo sido feita a escolha da forma geométrica da tremonha (cônica, no presente caso), determinados os ângulos de atrito com a parede e o ângulo de atrito interno efetivo do material, então, a máxima inclinação da tremonha (medida com relação a vertical), a qual assegura o fluxo de massa, já pode ser determinada.

A abordagem de JENIKE [6] para determinação do ângulo de inclinação da tremonha para fluxo mássico é baseado nas condições de equilíbrio aplicadas em um volume infinitesimal do material granulado na tremonha. Assim, Jenike determinou duas equações diferenciais parciais, cujas soluções existem apenas para combinações específicas dos parâmetros $\Theta_{\mathrm{c}}, \varphi_{\mathrm{e}}$, e $\varphi_{\mathrm{x}}$, ou seja, ângulo de inclinação, ângulo interno de atrito efetivo e ângulo de atrito com a parede, respectivamente. As combinações destes parâmetros podem prever o padrão de fluxo do material, se fluxo mássico ou fluxo de funil (Figura 8). Com essas combinações foram gerados diagramas para determinação do ângulo de inclinação para fornecer fluxo mássico ou de funil para diferentes ângulos de atrito interno efetivo e ângulo de atrito com a parede, como mostra a Figura 9. Jenike também recomenda, como fator de segurança, que o ângulo de inclinação da tremonha deve ser diminuído de $2^{\circ}$ a $5^{\circ}$ do que o determinado graficamente.
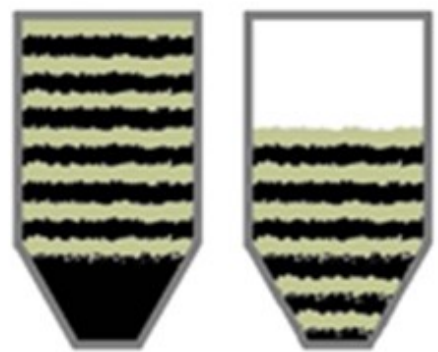

(a)

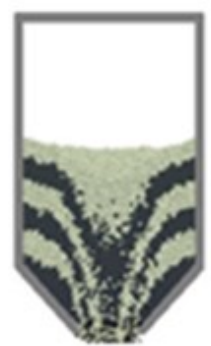

(b)

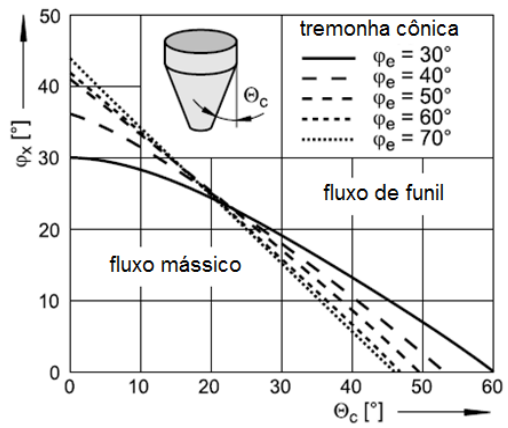

Figura 8: a) Fluxo másico. b) Fluxo de funil.

\subsection{Determinação do Diâmetro Mínimo para a descarga no silo}

Após a definição da inclinação da tremonha para fluxo mássico, faz-se necessário a determinação do valor do diâmetro mínimo para que não haja obstrução do fluxo por meio de formação de arcos coesivos. São três etapas a serem realizadas para o cálculo do diâmetro: i) determinação do fator de fluxo, ff, ii) determinação da tensão crítica, e finalmente, iii) o cálculo do diâmetro crítico.

Seja um arco estável hipotético, a tensão atuante neste arco é dada por $\sigma_{1}{ }^{\prime}[5,9]$, então se define fator de fluxo a razão $\mathrm{ff}=\sigma_{1} / \sigma_{1}{ }^{\prime}$. JENIKE [6] desenvolveu diagramas para a fácil determinação do fator de fluxo em função do ângulo de inclinação da tremonha, ângulo de atrito com a parede e ângulo de atrito interno efetivo, como mostra a Figura 10.

Existem outras metodologias para a determinação do fator de fluxo, tal como a teoria de ENSTAD [8], pela qual o ff é determinado da seguinte maneira [8,9]: primeiramente é definida a função $F\left(\Theta_{c}\right)$, que leva em consideração os efeitos da variação do ângulo de inclinação da tremonha e geometria da mesma:

$$
F\left(\Theta_{c}\right)=\left(\frac{65}{130+\Theta_{c}}\right)^{m}\left(\frac{200}{200+\Theta_{c}}\right)^{1-m}
$$

No qual m=1 para tremonhas cônicas. Então, o fator de fluxo é determinado por:

$$
\mathrm{ff}=\frac{\mathrm{Y}\left(1+\operatorname{sen} \varphi_{\mathrm{e}}\right)}{2(\mathrm{X}-1) \mathrm{F}\left(\Theta_{c}\right) \cos \left(90-\Theta_{c}\right)}
$$

sendo:

$$
\begin{aligned}
& 2 \beta=\varphi_{x}+\operatorname{sen}^{-1}\left(\frac{\operatorname{sen} \varphi_{x}}{\operatorname{sen} \varphi_{e}}\right) \quad X=\frac{2^{m} \operatorname{sen} \varphi_{e}}{1-\operatorname{sen} \varphi_{e}}\left[\frac{\operatorname{sen}(\beta+\alpha)}{\cos \left(90-\Theta_{c}\right)}+1\right] \quad \alpha=\beta+\Theta_{c} \\
& Y=\left[\frac{\left[2\left(1-\cos \Theta_{c}\right)^{m} \alpha^{1-m} \cos \left(90-\Theta_{c}\right)+\operatorname{sen} \beta(\operatorname{sen} \alpha)^{1+m}\right]}{\left(1-\operatorname{sen} \varphi_{e}\right)(\operatorname{sen} \alpha)^{2+m}}\right]
\end{aligned}
$$


Uma vez determinado o ff, determina-se a tensão inconfinada crítica no gráfico $\sigma_{\mathrm{c}} \mathrm{vs} \sigma_{1}$. Neste gráfico são inseridas as informações de FF e ff, ou seja, a curva de fator de fluxo FF e uma reta com ângulo de inclinação cuja tangente é o inverso de ff. Se no gráfico, a curva de FF ficar abaixo da reta de ff e as curvas não se interceptarem então a tensão desenvolvida em um arco coesivo no interior do silo é maior que a resistência do material, então sempre haverá fluxo e não há diâmetro mínimo para ser determinado. Se a curva de FF ficar acima da reta de ff e as curvas não se interceptarem, o material não escoará por gravidade e algum dispositivo extra no silo deverá ser usado forçar o fluxo. Se as curvas de FF e ff se interceptarem, este ponto de interseção define a tensão crítica no qual a tensão atuante no arco e a resistência são iguais (Figura 11).

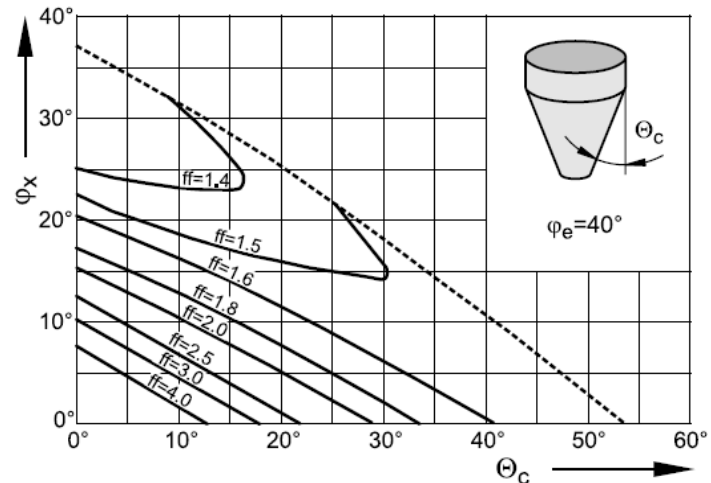

Figura 10: Diagrama para determinação de ff [5].

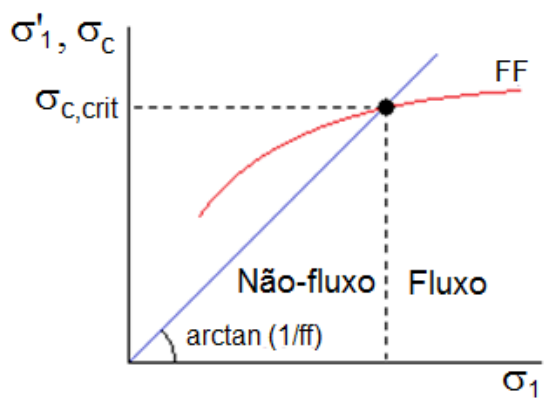

Figura 11: Determinação da tensão inconfinada crítica [9].

Após ter sido calculado o ff e determinado a tensão crítica $\left(\sigma_{c, \text { crit }}\right)$, o diâmetro crítico da tremonha cônica de um silo circular pode ser determinado por meio da seguinte equação $[5,6]$ :

$$
\mathrm{d}_{\text {crit }}=\frac{1000 \mathrm{H}\left(\Theta_{\mathrm{c}}\right) \sigma_{c, \text { crit }}}{9,81 \rho}
$$

sendo

$$
H=2+\frac{180 \Theta_{c}}{60 \pi}
$$

\section{RESULTADOS E DISCUSSÃO}

Os ensaios de cisalhamento em célula Jenike (Figura 12) foram realizados com tensões normais précisalhantes $\sigma_{\text {pré,1}}, \sigma_{\text {pré, } 2}=2 \sigma_{\text {pré,1 }}$ e $\sigma_{\text {pré,3 }}=4 \sigma_{\text {pré,1 }}$. Para cada tensão pré-cisalhante deve-se cisalhar novamente o material no mínimo 3 vezes com tensão normal na faixa de 80 a $40 \%$ da $\sigma_{\text {pré, }}$ correspondente. Segundo a norma ASTM [14], a tensão normal pré-cisalhante, $\sigma_{\text {pré, } 1}$ deve ser aproximadamente $2,5 \mathrm{kPa}$ para a densidade de $860 \mathrm{~kg} / \mathrm{m}^{3}$ do caulim. Os resultados obtidos para as tensões de pré-consolidação estão apresentados nas Tabelas 1 a 3 .
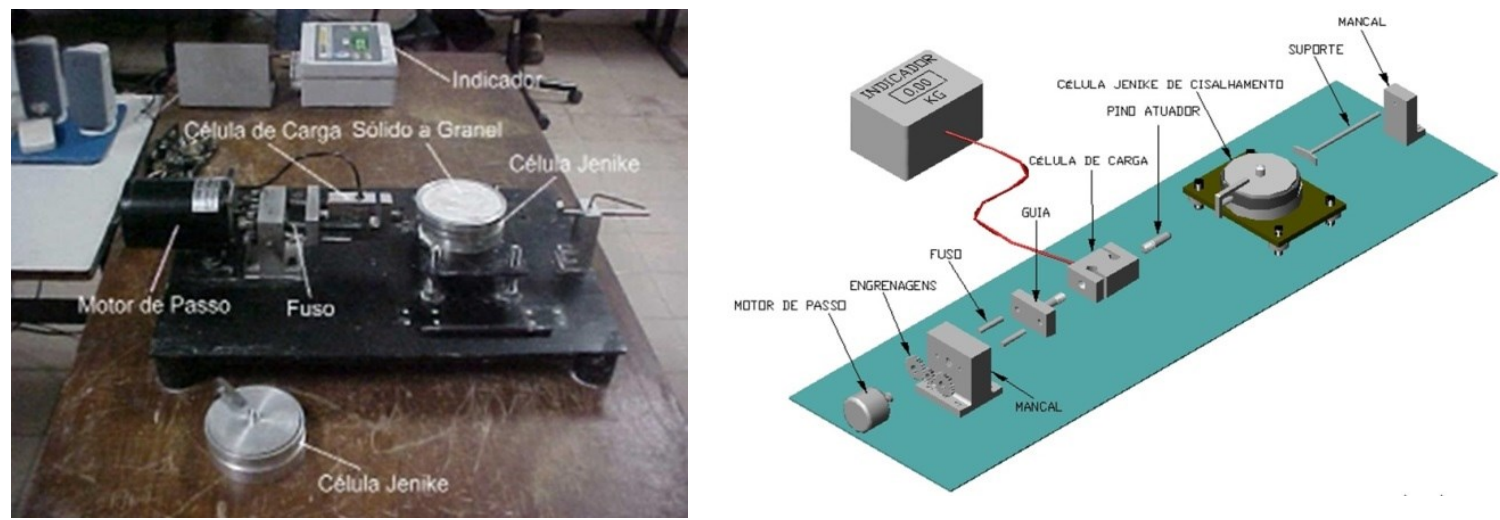

Figura 12: Visão geral e esquema do aparato experimental usando célula Jenike. 
Tabela 1: Ensaio de cisalhamento entre o caulim com $\sigma_{\text {pré, }}$.

\begin{tabular}{ccc}
\hline Etapa $\sigma_{\text {pré,1 }}$ & Tensão Normal $(\mathrm{kPa})$ & Tensão cisalhante $(\mathrm{kPa})$ \\
\hline Pré-cisalhamento & 2,518884 & 1,527 \\
Cisalhamento à aproximadamente $80 \%$ & 1,959132 & 1,455 \\
Cisalhamento à aproximadamente $60 \%$ & 1,539318 & 1,251 \\
Cisalhamento à aproximadamente $40 \%$ & 1,119504 & 1,058 \\
\hline
\end{tabular}

Tabela 2: Ensaio de cisalhamento entre o caulim com $\sigma_{\text {pré,2. }}$.

\begin{tabular}{ccc}
\hline Etapa $\sigma_{\text {pré, }}$ & Tensão Normal $(\mathrm{kPa})$ & Tensão cisalhante $(\mathrm{kPa})$ \\
\hline Pré-cisalhamento & 5,037769 & 2,844 \\
Cisalhamento à aproximadamente $80 \%$ & 3,918265 & 2,606 \\
Cisalhamento à aproximadamente $60 \%$ & 2,938698 & 2,323 \\
Cisalhamento à aproximadamente $40 \%$ & 2,09907 & 1,856 \\
\hline
\end{tabular}

Tabela 3: Ensaio de cisalhamento entre o caulim com $\sigma_{\text {pré, } 3 .}$

\begin{tabular}{ccc}
\hline Etapa $\sigma_{\text {pré,3 }}$ & Tensão Normal $(\mathrm{kPa})$ & Tensão cisalhante $(\mathrm{kPa})$ \\
\hline Pré-cisalhamento & 10,07554 & 5,649 \\
Cisalhamento à aproximadamente $80 \%$ & 7,976467 & 5,004 \\
Cisalhamento à aproximadamente $60 \%$ & 6,017335 & 4,332 \\
Cisalhamento à aproximadamente $40 \%$ & 4,058203 & 3,496 \\
\hline
\end{tabular}

Seguindo as considerações dadas em SCHULZE [5] e na norma ASTM [14] realizou-se um equacionamento para a obtenção dos círculos de Mohr. Foi feito um programa computacional por meio do software MATLAB para determinar as tensões normais principais $\sigma_{1}$ e $\sigma_{2}$, ângulo de atrito interno efetivo $\varphi_{e}$ e tensão inconfinada $\sigma_{\mathrm{c}}$ para cada ensaio. Os resultados obtidos estão apresentados na Tabela 4 e o círculo de Mohr para o ensaio com a $1^{\underline{a}}$ tensão de pré-consolidação está mostrado na Figura 13.

Tabela 4: Tensões normais (em kPa), ângulo de atrito interno e escoabilidade obtidos.

\begin{tabular}{|c|c|c|c|c|c|c|c|c|c|c|c|c|c|c|}
\hline \multicolumn{5}{|c|}{$1^{o}$ Ensaio $\left(\sigma_{\text {pré, }}\right)[(\mathrm{kPa})]$} & \multicolumn{5}{|c|}{$2^{\mathrm{o}}$ Ensaio $\left(\sigma_{\text {pré }, 2}\right)[(\mathrm{kPa})$} & \multicolumn{5}{|c|}{$3^{\circ}$ Ensaio $\left(\sigma_{\text {pré } 3}\right)[(\mathrm{kPa})$} \\
\hline$\sigma_{1}$ & $\sigma_{2}$ & $\sigma_{\mathrm{c}}$ & $\varphi_{\mathrm{e}}$ & $\mathrm{ff}_{\mathrm{c}}$ & $\sigma_{1}$ & $\sigma_{2}$ & $\sigma_{\mathrm{c}}$ & $\varphi_{\mathrm{e}}$ & $\mathrm{ff}_{\mathrm{c}}$ & $\sigma_{1}$ & $\sigma_{2}$ & $\sigma_{\mathrm{c}}$ & $\varphi_{\mathrm{e}}$ & $\mathrm{ff}_{\mathrm{c}}$ \\
\hline 3,96 & 0,90 & 1,66 & $38,99^{\circ}$ & 2,39 & 7,90 & 2,21 & 3,10 & $34,21^{\circ}$ & 2,55 & 16,34 & 4,98 & 5,71 & $32,18^{\circ}$ & 2,86 \\
\hline
\end{tabular}

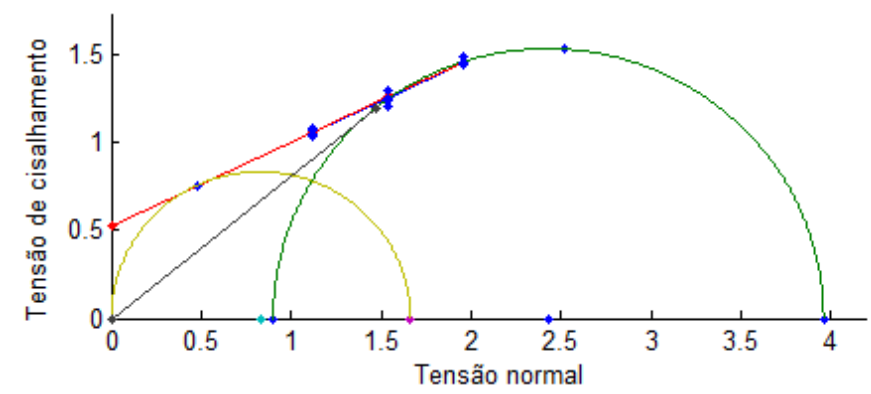

Figura 13: Círculo de Mohr para a primeira tensão de pré-consolidação. 
De acordo com os resultados de $\sigma_{1}$ e $\sigma_{c}$, a curva do fator de fluxo FF foi determinada (Figura 14). Com a curva de FF obtida, pode-se avaliar qual o grau de escoabilidade do caulim. SCHULZE [5] apresenta uma medida da escoabilidade (flowability, $\mathrm{ff}_{\mathrm{c}}=\sigma_{1} / \sigma_{\mathrm{c}}$ ) que indica o quanto o material é facilmente escoável (Tabela 5). Desta forma, pode-se classificar o caulim com 4\% de umidade como sendo um material coesivo, observando os valores escoabilidade na Tabela 4 e também pelo formato da curva FF. Quanto mais a curva de FF se aproxima da horizontal, maior a escoabilidade (razão $\sigma_{1} / \sigma_{\mathrm{c}}$ ) do material e mais fácil será o fluxo (Figura 15) [15]. Conforme Figura 14, a curva de FF não tem um padrão de fácil escoamento.

Tabela 5: Faixa de valores da escoabilidade $\left(\mathrm{ff}_{\mathrm{c}}\right)$ e classificação do material quanto ao seu fluxo [5].

\begin{tabular}{ccc}
\hline Faixa de $\mathrm{ff}_{\mathrm{c}}$ & & Material em relação ao escoamento \\
\cline { 1 - 1 } $\mathrm{ff}_{\mathrm{c}}<1$ & & Material não escoa \\
$1<\mathrm{ff}_{\mathrm{c}}<2$ & & Material muito coesivo \\
$2<\mathrm{ff}_{\mathrm{c}}<4$ & & Material coesivo de fácil escoamento \\
$4<\mathrm{ff}_{\mathrm{c}}<10$ & & Material com escoamento livre \\
$10<\mathrm{ff}_{\mathrm{c}}$ &
\end{tabular}

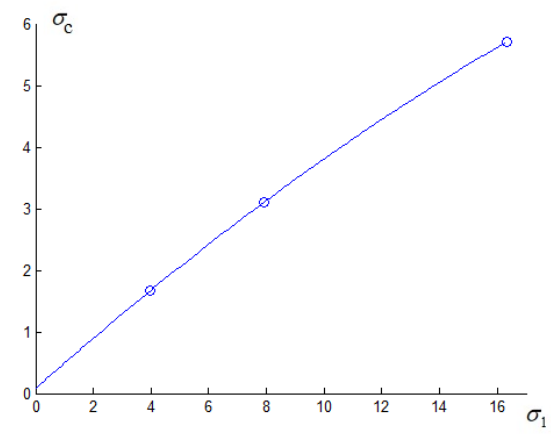

Figura 14: Função FF obtida para o caulim.

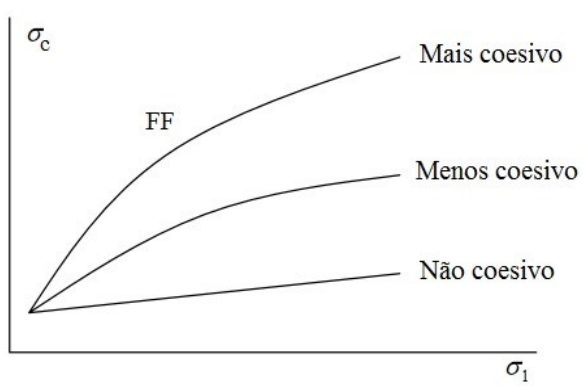

Figura 15: Formatos de curvas FF e coesividade [15].

De acordo com os dados dos círculos de Mohr, o ângulo de atrito interno efetivo foi encontrado a partir da melhor reta partindo da origem através dos pontos $E_{i}$ de cada tensão pré-cisalhante (Figura 16).

Os testes de cisalhamento caulim-parede (caulim-acrílico) forneceram dados para formar o gráfico da Figura 17 e resultaram em:

- $\quad$ Ângulo de atrito com a parede $\left(\varphi_{\mathrm{x}}\right)=21,6^{\circ}$

- $\quad$ Coeficiente de atrito com a parede $(\mu)=0.40$

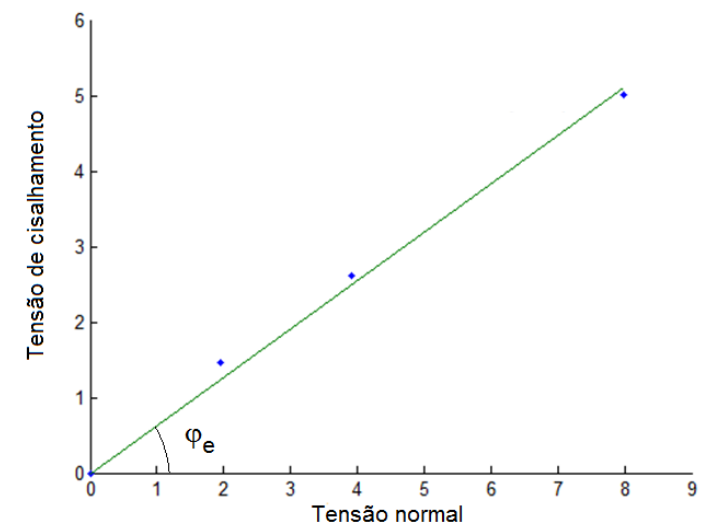

Figura 16: Reta para determinação do ângulo de atrito interno efetivo.

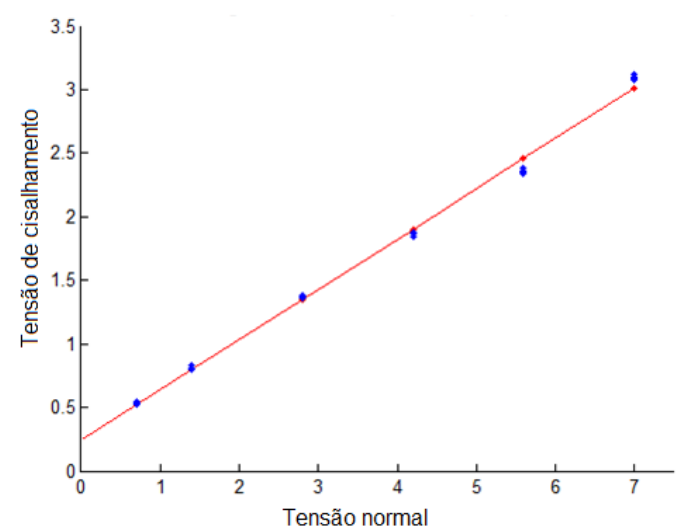

Figura 17: Reta para determinação do ângulo de atrito com a parede. 
Por meio da teoria de JENIKE [6], com o ângulo de atrito efetivo $32,6^{\circ}$ e ângulo de atrito com parede de $21,6^{\circ}$, a inclinação limite da tremonha fica em aproximadamente $26^{\circ}$ para fornecer fluxo mássico. Jenike sugere que a inclinação seja diminuída em 3 a $5^{\circ}$ como fator de segurança, e assim o ângulo de inclinação da tremonha com a vertical ficou em $21^{\circ}$ (Figura 18).

Depois de encontrado a inclinação da tremonha, faz-se necessário encontrar o fator de fluxo, para possibilitar encontrar a tensão crítica e assim calcular o diâmetro mínimo necessário para evitar obstruções. O valor de $f f$ foi encontrado tanto pela teoria de JENIKE [6] quanto por ENSTAD [8].

$\mathrm{O}$ valor do fator de fluxo pela teoria de Jenike foi encontrado ser igual a 1,8 e o gráfico do fator de fluxo (ff) x função fluxo (FF) forneceu uma tensão crítica de $0,317 \mathrm{kPa}$ (Figura 19).

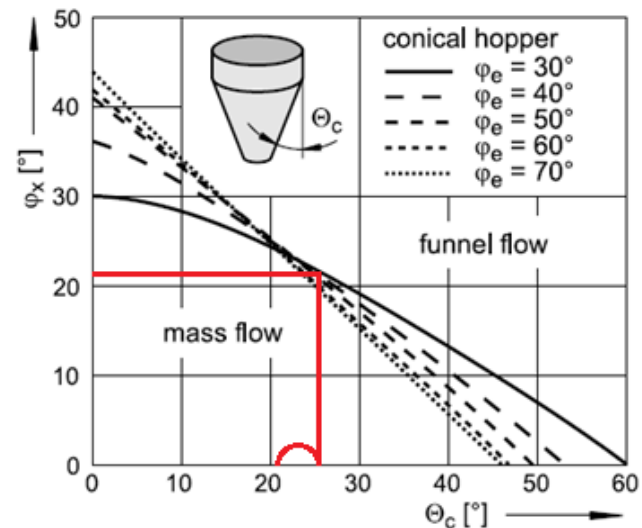

Figura 18: Gráfico de Jenike para encontrar a inclinação da tremonha para fluxo mássico.

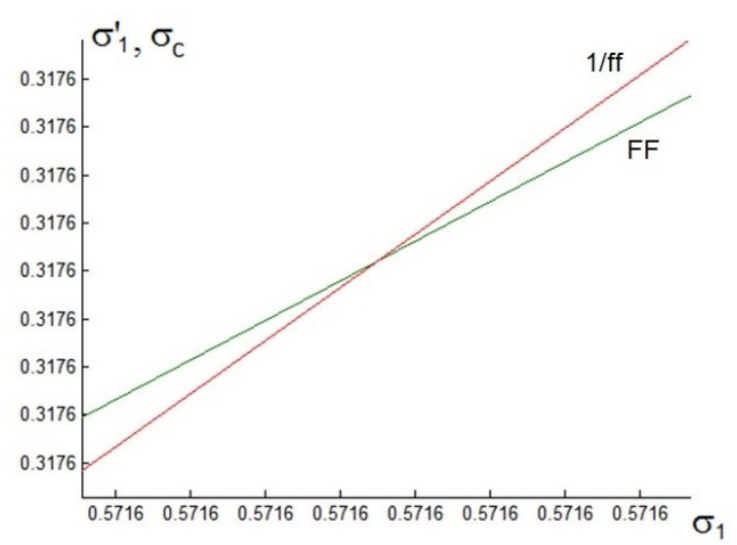

Figura 19: Determinação da tensão crítica pelo gráfico do Fator de fluxo $(f f) \times$ função fluxo $(\mathrm{FF})$.

De acordo com as formulações de ENSTAD [8], obteve-se um fator de fluxo igual a 1,46 e por meio do gráfico fator de fluxo $(f f) \times$ função fluxo $(\mathrm{FF})$ a tensão crítica resultou em $0,205 \mathrm{kPa}$. Com os valores de tensões críticas obtidos pelas duas teorias, foram determinados os respectivos diâmetros críticos usando as equações (6) e (7), que resultaram nos valores apresentados na Tabela 6. De acordo com esses valores, podese verificar que a teoria de JENIKE [6] mais conservadora que a de ENSTAD [8].

Tabela 6: Resultados das teorias de JENIKE [6] e ENSTAD [8].

\begin{tabular}{ccc}
\hline Resultados das teorias & Tensão crítica & Diâmetro crítico \\
\hline JENIKE [6] & $0,317 \mathrm{kPa}$ & $8,8 \mathrm{~cm}$ \\
ENSTAD [8] & $0,205 \mathrm{kPa}$ & $5,7 \mathrm{~cm}$ \\
\hline
\end{tabular}

Nos ensaios experimentais em silo, foram utilizadas 7 tremonhas com diferentes inclinações e diâmetros de saída: uma tremonha com $18^{\circ}$ de inclinação e $10 \mathrm{~cm}$ de diâmetro de saída; uma tremonha com $21^{\circ}$ de inclinação e $8,7 \mathrm{~cm}$ de saída; quatro tremonhas com $24^{\circ}$ de inclinação e com diâmetros de $1,40 \mathrm{~cm}, 3,40 \mathrm{~cm}$, $4,0 \mathrm{~cm}$ e $4,70 \mathrm{~cm}$, respectivamente, e uma tremonha com $31^{\circ}$ de inclinação com $9 \mathrm{~cm}$ de diâmetro na saída. A Figura 20 mostra um desses ensaios, que foi o escoamento de caulim no silo com tremonha de $18^{\circ}$ de inclinação e $10 \mathrm{~cm}$ de diâmetro, que forneceu um fluxo mássico. Os resultados dos ensaios para todas as tremonhas estão apresentados na Tabela 7, pela qual se pode verificar que as teorias conseguiram prever o tipo de fluxo e se haveria obstruções.

Tabela 7: Resultado dos ensaios de escoamento nos silos.

\begin{tabular}{cccc}
\hline Inclinação da tremonha & Diâmetro de saída & Obstrução & Fluxo obtido experimentalmente \\
\hline $18^{\circ}$ & $10 \mathrm{~cm}$ & Não & Fluxo mássico \\
$21^{\circ}$ & $8,8 \mathrm{~cm}$ & Não & Fluxo mássico \\
$24^{\circ}$ & $1,4 \mathrm{~cm}$ & Sim & Sem escoamento \\
$24^{\circ}$ & $3,4 \mathrm{~cm}$ & Sim & Sem escoamento \\
$24^{\circ}$ & $4,0 \mathrm{~cm}$ & Sim & Sem escoamento \\
$24^{\circ}$ & $4,7 \mathrm{~cm}$ & Sim & Sem escoamento \\
$31^{\circ}$ & $9,0 \mathrm{~cm}$ & Não & Fluxo funil \\
\hline
\end{tabular}



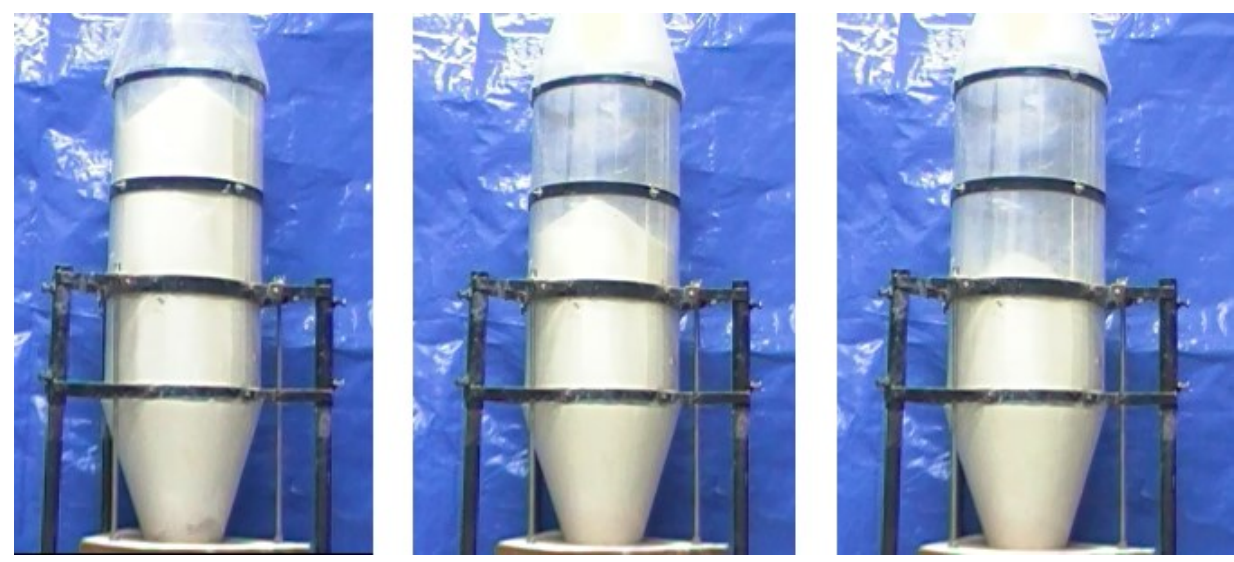

Figura 20: Fluxo de caulim no silo com tremonha de 18 graus com $10 \mathrm{~cm}$ de abertura de descarga.

\section{CONCLUSÕES}

O trabalho focou na identificação das propriedades de fluxo do caulim no dimensionamento de tremonha cônica de silo de acrílico para escoamento de caulim para fluxo mássico, por meio das teorias de JENIKE [6] e ENSTAD [8], e também realização de ensaios experimentais com diferentes tremonhas de acrílico para comprovação dos resultados teóricos. O material acrílico foi escolhido para o silo visando possibilitar a visualização do padrão de fluxo desenvolvido.

De acordo com os resultados obtidos verificou-se que o caulim com $4 \%$ de umidade é um material granulado classificado como coesivo, não possuindo um fluxo livre. Portanto, faz-se necessário um dimensionamento criterioso de tremonhas de silos para o armazenamento e descarga desse material para que não ocorram problemas tais como entupimentos, segregação, dentre outros. Assim, foi verificado que para o ângulo de inclinação de tremonha cônica, a teoria de Jenike resulta em um ângulo de inclinação com a vertical de $21^{\circ}$. Em relação ao diâmetro de descarga da tremonha, verificou-se teoricamente que a teoria de JENIKE [6] é mais conservadora que a teoria de ENSTAD [8]. No primeiro caso, encontrou-se um valor crítico de 8,8 $\mathrm{cm}$, enquanto que por Enstad o valor do diâmetro crítico encontrado foi $5,7 \mathrm{~cm}$.

Nos experimentos de escoamento nos silos acrílicos em laboratório, foram obtidos os padrões de fluxo mássico para tremonhas de até $21^{\circ}$, enquanto que para angulações maiores houve escoamento com fluxo de funil, quando não sofreram arqueamento. Houve arqueamento para diâmetros de descarga abaixo de $5,7 \mathrm{~cm}$, já para diâmetros acima de $8,8 \mathrm{~cm}$ não houve a formação de arcos coesivos. Portanto, os ensaios em laboratório estiveram de acordo com a teoria. Contudo, não foi possível realizar experimentos com tremonhas com diâmetros que ficassem entre os valores mínimos das duas teorias, que possibilitasse verificar experimentalmente o conservadorismo da teoria de Jenike no cálculo do diâmetro mínimo que evite o arqueamento. Isso motivou o uso do método dos elementos discretos para simulação virtual do escoamento, cujos resultados serão apresentados em trabalhos futuros.

\section{AGRADECIMENTOS}

Os autores agradecem à FAPESPA (Fundação Amazônia de Amparo a Estudos e Pesquisa do Pará) pelo suporte financeiro.

\section{BIBLIOGRAFIA}

[1] PAIVA, R., CARNEIRO, C., CUNHA, E., et al., "Lavra da mina de caulim de Ipixuna do Pará da Imerys RCC", In: Anais do $8^{\underline{o}}$ Congresso Brasileiro de Mina a Céu Aberto, Escola de Engenharia da UFMG, Belo Horizonte/MG, 6 a 8 de agosto, 2014.

[2] SANTOS, L., MELCHIADES, F., BISCARO, E., et al., "Avaliação de caulim sedimentar do Estado do Pará como matéria-prima para o setor cerâmico. Parte I. Caracterização físico-química”, Cerâmica Industrial, v. 15, n. 5-6, Setembro/Dezembro, 2010.

[3] MENEZES, R., ALMEIDA, R., SANTANA, L., et al., "Utilização do resíduo do beneficiamento do caulim na produção de blocos e telhas cerâmicos”, Revista Matéria, v. 12, n. 1, pp. 226-236, 2007.

[4] Departamento Nacional de Produção Mineral, SUMÁRIO MINERAL 2014, http://www.dnpm.gov.br/dnpm/sumarios/sumario-mineral-2014. Acessado em agosto de 2016. 
[5] SCHULZE, D., Powders and bulk solids - behavior, characterization, storage and flow, Springer, 2007. [6] JENIKE, A., Storage and flow of solids, Bulletin 123, University of Utah Engineering Station, 1964 (revised, 1976).

[7] WALKER, D. “An approximate theory for pressures and arching in hoppers", Chemical \& Engineering Sciences, v. 21, pp. 975-997, 1966.

[8] ENSTAD, G. “On the theory of arching in mass flow hoppers”, Chemical \& Engineering Science, v. 30, pp. 1273-1283, 1975.

[9] CALIL JUNIOR, C., CHEUNG, A. Silos: pressões, fluxo, recomendações de para o projeto e exemplos de cálculo, Editora da Escola de Engenharia de São Carlos - USP, 2007.

[10] LOPES NETO, J., NASCIMENTO, J., SILVA, R., et al., "Powder flow criteria for design of vertical silo walls", Engenharia Agrícola, v.33, n.3, pp.453-462, 2013.

[11] GUO, J., ROBERTS, A., PRIGGE, J. "The Investigation of Arch Model Acting in Mass-Flow Hoppers", Advanced Materials Research, v. 508, pp. 135-140, 2012.

[12] GUO, J., ROBERTS, A., PRIGGE, J., "Experimental investigation of wall pressure and arching behavior under surcharge pressure in mass-flow hoppers", Powder Technology, v. 258, pp.272-284, 2014.

[13] IMERYS, http://www.imerysnopara.com.br. Acessado em setembro de 2016.

[14] ASTM, Standard D6128-97, Standard Test Method for Shear Testing of Bulk Solids Using the Jenike Shear Cell, American Society for Testing and Materials, 1997.

[15] CHASE, G., http://www.inti.gob.ar/cirsoc/pdf/silos/SolidsNotes10HopperDesign.pdf. Acessado em setembro de 2016.

[16] CHAVES, A., FERREIRA, F., LIMA, J., et al., Teoria e prática do tratamento de minérios: manuseio de sólidos granulados, Editora Signus, 2011.

[17] MCGLINCHEY, D. Bulk solids handling - Equipment selection and operation. Blackwell Publishing, 2008 .

[18] MEHOS, G., MORGAN, D., HOPPER DESIGN PRINCIPLES, http://www.chemengonline.com/hopper-design-principles/?printmode=1. Acessado em setembro de 2016. 